\title{
On the applicability of local softness and hardness
}

\author{
M. Torrent-Sucarrat, ${ }^{a b}$ F. De Proft, ${ }^{a}$ P. W. Ayers $^{c}$ and P. Geerlings ${ }^{a}$ \\ Received 18th September 2009, Accepted 29th October 2009 \\ First published as an Advance Article on the web 3rd December 2009 \\ DOI: $10.1039 /$ b919471a
}

Global hardness and softness and the associated hard/soft acid/base (HSAB) principle have been used to explain many experimental observed reactivity patterns and these concepts can be found in textbooks of general, inorganic, and organic chemistry. In addition, local versions of these reactivity indices and principles have been defined to describe the regioselectivity of systems. In a very recent article (Chem.-Eur. J. 2008, 14, 8652), the present authors have shown that the picture of these well-known descriptors is incomplete and that the understanding of these reactivity indices must be "reinterpreted". In fact, the local softness and hardness contain the same "potential information" and they should be interpreted as the "local abundance" or "concentration" of their corresponding global properties. In this contribution, we analyze the implications of this new point of view for the applicability of these well-known descriptors when comparing two sites in three situations: two sites within one molecule, two sites in two different, but noninteracting molecules, and two sites in two different, but interacting, molecules. The implications on the HSAB principle are highlighted, leading to the discussion of the role of the electrostatic interaction.

\section{Introduction}

One of the main objectives of chemistry is to offer in an easy and elegant way to explain very complicated processes. Over the past decades theoretical chemists have provided tools (concepts and principles) for understanding very complex processes not only for their community, but also to scientists working in neighbouring fields (biology, materials science,...). The work by Pearson on chemical hardness and softness and the hard and soft acids and bases principle (HSAB) ${ }^{1-6}$ is an exemplar for this approach and its success. The HSAB principle asserts that hard acids preferentially coordinate with hard bases, while soft acids prefer to react with soft bases, from both the thermodynamic and kinetic points of view. In this classification, soft systems are large and highly polarizable, while hard systems are relatively small and much less polarizable.

Conceptual density functional theory (DFT) ${ }^{7}$ tries to play the same role in chemistry as chemistry does for the other sciences. The reactivity descriptors and principles rationalized within the framework of the conceptual $\mathrm{DFT}^{7-13}$ have allowed an easy understanding of many organic, inorganic, and biochemical reactions. However, one should always be careful not to use these indices and principles beyond their range of applicability; otherwise contradictory results are sometimes generated and skepticism about the utility of these concepts may arise. The aim of this work is to present a recipe for the

\footnotetext{
${ }^{a}$ Member of the QCMM Alliance group Ghent/Brussels, Eenheid Algemene Chemie (ALGC), Faculteit Wetenschappen, Vrije Universiteit Brussel (VUB), Pleinlaan 2, 1050 Brussels, Belgium.E-mail: mtorrent@vub.ac.be

${ }^{b}$ Departament de Química Biologica i Modelització Molecular. IQAC-CSIC, c/ Jordi Girona 18, E-08034 Barcelona, Spain

${ }^{c}$ Department of Chemistry, McMaster University, Hamilton, Ontario, L8S4M1, Canada
}

correct application of the well-known, but sometimes poorly understood and misused, concepts of local softness and hardness.

The global hardness, $\eta,{ }^{14}$ given as the second derivative of the energy, $E$, with respect to the number of electrons, $N$, at constant external potential, $\nu(\vec{r})$, captures the resistance of a chemical species to changing its electronic number

$$
\eta=S^{-1}=\left(\frac{\partial^{2} E}{\partial N^{2}}\right)_{\nu(\vec{r})}=\left(\frac{\partial \mu}{\partial N}\right)_{\nu(\vec{r})} .
$$

Here $S$ and $\mu$ denote the global softness and the chemical potential, respectively. These indices are called global descriptors because they describe the properties of a molecule as a whole. To be the preferred site for a chemical reaction, a local HSAB principle was proposed and the local counterparts of softness and hardness were introduced: local softness, $s(\vec{r}),{ }^{15}$ and hardness, $\eta(\vec{r}),{ }^{16,17}$ defined as

$$
\begin{aligned}
& \eta(\vec{r})=\left(\frac{\delta \mu}{\delta \rho(\vec{r})}\right)_{v(\vec{r})} \text { and } \\
& s(\vec{r})=\left(\frac{\delta \rho(\vec{r})}{\delta \mu}\right)_{v(\vec{r})} .
\end{aligned}
$$

The local hardness and local softness are interconnected through the following inverse relationship

$$
\int \eta(\vec{r}) s(\vec{r}) \mathrm{d} \vec{r}=1 .
$$

Notice that eqn (4) is not directly connected to the inverse relationship between the global hardness and global softness, eqn (1); $\eta(\vec{r})$ is not equal to $1 / s(\vec{r})$. The local hardness defined by eqn (2) is, however, ambiguous, ${ }^{17-21}$ because the constraint of the external potential, $\nu(\vec{r})$, is not required to define the variations of the chemical potential with respect to the electron 
density. This ambiguity has been circumvented in three different ways: the frontier local hardness, ${ }^{19,22-29}$ the total local hardness, ${ }^{17,19,30-39}$ and the unconstrained local hardness. ${ }^{40}$ In this work, we will focus on the frontier local hardness, ${ }^{41}$ which is defined as

$$
\eta(\vec{r})=\int \frac{\delta^{2} F[\rho(\vec{r})]}{\delta \rho(\vec{r}) \delta \rho\left(\vec{r}^{\prime}\right)} f\left(\vec{r}^{\prime}\right) \mathrm{d} \vec{r}^{\prime}=\int \eta\left(\vec{r}, \vec{r}^{\prime}\right) f\left(\vec{r}^{\prime}\right) \mathrm{d} \vec{r}^{\prime},
$$

where $F[\rho(\vec{r})]$ is the universal Hohenberg-Kohn functional and $\eta\left(\vec{r}, \vec{r}^{\prime}\right)$ is the hardness kernel, ${ }^{17,42}$ the second functional derivative of $F[\rho(\vec{r})]$ with respect to the density.

In a very recent article, ${ }^{43}$ the present authors show that the largest values of the $s(\vec{r})$ and $\eta(\vec{r})$ do not necessarily correspond to the softest and hardest regions of the molecule, respectively. Moreover, we present there that the only acceptable interpretation for the local softness and local hardness is that these functions are pointwise measures of the "local abundance" or "concentration" of the corresponding global quantities. In this framework, $s(\vec{r})$ and $\eta(\vec{r})$ contain the same potential "information" and they become more general because they can be applied both to hard and soft systems. In a soft system $s(\vec{r})$ and $\eta(\vec{r})$ both describe the soft site of a molecule, while in a hard system $s(\vec{r})$ and $\eta(\vec{r})$ both describe the hard site of the molecule. In the present work, we will analyze the implications of this new perspective in the applicability of these well-known local reactivity indicators.

In contrast to the global HSAB principle, the quantitative representation of the local $\mathrm{HSAB}$ principle has not been an easy task. Klopman already proposed in 1968 that the soft-soft interactions are frontier-controlled and predominantly covalent in nature. ${ }^{44}$ Then the preferred site of the molecule to react in a soft-soft interaction is the region with the maximum value of $f(\vec{r}) .^{45-48}$ On the contrary, the conditions for the chargecontrolled and ionic hard-hard interactions are not so clear in the literature and even contradictory results are obtained. $\mathrm{Li}$ and Evans ${ }^{47}$ propose that hard systems show preferential reactivity at the site where $f(\vec{r})$ is a minimum and different applications following this line have been reported. ${ }^{46,49,50}$ However, Chattaraj and collaborators ${ }^{51,52}$ argue that hard-hard interactions are predominantly ionic in nature and charge-controlled and that for these reactions the preferred site is the one with maximum net charge, in certain cases coinciding with the site with the minimum value of the Fukui function. Moreover, some of the present authors ${ }^{37,53-55}$ have proposed schemes for a combined reactivity indicator, one component being appropriate for soft reactive sites (associated with frontier-orbital control) and another appropriate for hard reactive sites (associated with electrostatic control). In this way, using the correct choice of the weight factors for these two reactivity descriptors, one can describe any acid-base reaction. The present work provides a different viewpoint based on the new interpretation of $s(\vec{r})$ and $\eta(\vec{r})$.

\section{Theoretical background and computational details}

Starting with the global descriptors, the two most popular operational equations of the global hardness and softness are obtained applying the finite difference approximation to eqn (1)

$$
\eta=S^{-1} \cong I-A \text { and }
$$

introducing Koopmans' theorem ${ }^{56}$

$$
\eta=S^{-1} \cong \varepsilon_{\text {LUMO }}-\varepsilon_{\text {HOMO }}
$$

Here $I$ and $A$ are the first vertical ionization potential and electron affinity of the reference $N$-electron system in most cases a neutral molecule, respectively, and $\varepsilon_{\mathrm{HOMO}}$ and $\varepsilon_{\mathrm{LUMO}}$ are the energies of the highest occupied molecular orbital (HOMO) and the lowest unoccupied molecular orbital (LUMO), respectively. To evaluate $s(\vec{r})$, one can use the following chain rule

$$
s(\vec{r})=\left(\frac{\delta \rho(\vec{r})}{\delta \mu}\right)_{v(\vec{r})}=\left(\frac{\partial \rho(\vec{r})}{\partial N}\right)_{v(\vec{r})}\left(\frac{\delta N}{\delta \mu}\right)_{v(\vec{r})}=f(\vec{r}) S,
$$

where $f(\vec{r})$ is the Fukui function, a normalized function, which measures the propensity of a reagent to accept (or donate) electrons from (to) another chemical system. ${ }^{57,58}$ Notice that local softness is normalized to the global softness. For a molecular or atomic system, the derivative of $\rho(\vec{r})$ with respect to the number of electrons is discontinuous. This led Parr and Yang ${ }^{57}$ to identify the left, $f^{-}(\vec{r})$, and right, $f^{+}(\vec{r})$, derivatives as reactivity indices for electrophilic and nucleophilic attacks on the system, respectively. By applying a finite difference approximation and the frontier-electron theory of reactivity as formulated by Fukui and collaborators, ${ }^{59,60}$ the Fukui functions approximations can be written as

$$
\begin{aligned}
& f^{-}(\vec{r}) \cong \rho_{N}(\vec{r})-\rho_{N-1}(\vec{r}) \cong \rho_{\mathrm{HOMO}}(\vec{r})=\left|\phi_{\mathrm{HOMO}}(\vec{r})\right|^{2} \\
& \text { and } \\
& f^{+}(\vec{r}) \cong \rho_{N+1}(\vec{r})-\rho_{N}(\vec{r}) \cong \rho_{\mathrm{LUMO}}(\vec{r})=\left|\phi_{\mathrm{LUMO}}(\vec{r})\right|^{2},
\end{aligned}
$$

where $\rho_{N}(\vec{r}), \rho_{N-1}(\vec{r})$, and $\rho_{N+1}(\vec{r})$ are the electronic densities of the system with $N, N-1$, and $N+1$ electrons, respectively, and $\rho_{\mathrm{HOMO}}(\vec{r})$ and $\rho_{\mathrm{LUMO}}(\vec{r})$ are the densities of the HOMO and LUMO orbitals, respectively. Applying eqn (9) and (10) to $s(\vec{r})$, one obtains the electrophilic and nucleophilic local softnesses

$$
s^{x}(\vec{r})=f^{x}(\vec{r}) S,
$$

where $x$ can be + and - . Moreover, Yang and Mortier propose $^{61}$ that the condensed local Fukui, $f_{i}^{x}$, and condensed local softness, $s_{i}^{x}$, can be evaluated through integration of eqn (9)-(11) over atomic regions

$$
s_{i}^{x}=f_{i}^{x} S=S \int_{\Omega_{i}} f_{i}^{x}(\vec{r}) \mathrm{d} \vec{r} .
$$

These integrals can be evaluated using different population analysis techniques, e.g. Mulliken, ${ }^{62}$ natural population analysis (NPA), ${ }^{63}$ or integration over atomic domains. ${ }^{64,65}$ In the literature, one can find many different techniques for decomposing the molecular space into atomic domains, e.g. Atoms in molecules ${ }^{66}$ and Hirshfeld. ${ }^{67}$ In this work, we will use the fuzzy Voronoi polyhedra. ${ }^{68} \mathrm{~A}$ Voronoi polyhedron for an nucleus $i$ of a molecule is defined as the region enclosed by 
all the perpendicular planes to the vector joining $i$ to the other nuclei of the system. A fuzzy Voronoi polyhedron has the value unity in the vicinity of its own nucleus, but vanishes in a continuous and well-behaved manner near any other nucleus.

The local hardness can be evaluated through eqn (5), but approximations to the universal Hohenberg-Kohn functional and the Fukui function are required. ${ }^{69}$ As we have done in our previous articles, ${ }^{27,43}$ the hardness kernel has been approximated using the second order derivative of the Coulombic Thomas-Fermi-1/9th Weizsäcker-Dirac-Wigner functional with respect to the density. As $s(\vec{r})$ and $\eta(\vec{r})$ contain the same potential information, it is possible to write the analogue of eqn (11) in the local hardness and to define its electrophilic and nucleophilic counterparts ${ }^{32}$

$$
\eta^{x}(\vec{r})=\eta(\vec{r})\left[f^{x}\left(\vec{r}^{\prime}\right)\right]=\int \eta\left(\vec{r}, \vec{r}^{\prime}\right) f^{x}\left(\vec{r}^{\prime}\right) \mathrm{d} \vec{r}^{\prime} .
$$

In this text, we will not use the superscript for the local hardness to define the left and right derivatives. It will be represented between brackets with the approximation used to evaluate the Fukui function in eqn (13). For instance, $\eta(\vec{r})\left[\rho_{\mathrm{HOMO}}\left(\vec{r}^{\prime}\right)\right]$ will refer to $\eta^{-}(\vec{r})$ using $\rho_{\mathrm{HOMO}}\left(\vec{r}^{\prime}\right)$ to approximate $f^{-}\left(\vec{r}^{\prime}\right)$. Moreover, the global hardness can be obtained from the local hardness

$$
\eta=\int f(\vec{r}) \eta(\vec{r}) \mathrm{d} \vec{r}=\iint f(\vec{r}) \eta\left(\vec{r}, \vec{r}^{\prime}\right) f\left(\vec{r}^{\prime}\right) \mathrm{d} \vec{r} \mathrm{~d} \vec{r}^{\prime},
$$

where it is again necessary to approximate the Fukui function. We will use the nomenclature $\eta\left[\rho_{\mathrm{HOMO}}(\vec{r})\right]$ and $\eta\left[\rho_{\mathrm{LUMO}}(\vec{r})\right]$ to refer to the global hardnesses evaluated from eqn (14) and (5) using the $\rho_{\mathrm{HOMO}}(\vec{r})$ and $\rho_{\mathrm{LUMO}}(\vec{r})$, respectively, as approximations of $f(\vec{r})$. In analogy to the condensed local softness, it is possible to integrate eqn (14) over atomic regions, obtaining the condensed atomic hardness ${ }^{27}$

$$
\eta_{i}=\int_{\Omega_{i}} \eta(\vec{r}) f(\vec{r}) \mathrm{d} \vec{r} .
$$

All calculations were carried with $6-31+\mathrm{G}(\mathrm{d})$ basis sets $^{70,71}$ at the B3LYP ${ }^{72,73}$ level using the Gaussian03 package. ${ }^{74}$ The calculations have been done within the restricted formalism except for open-shell systems, where the unrestricted approach has been used. As in our previous works, ${ }^{26,27,75}$ the integrals of eqn (12) and (15) have been evaluated numerically using the Becke's multicenter integration scheme, ${ }^{68}$ which has been implemented in a program developed in our laboratory. This integration scheme decomposes the integration of a function over the $3 \mathrm{D}$ space into a sum of integrations over single-atom components using a weight function, $w_{i}(\vec{r})$, which has the value 1 in the vicinity of its own nucleus, but vanishes in a continuous and well-behaved manner near any other nucleus. The $w_{i}(\vec{r})$ used in this work is the fuzzy Voronoi polyhedra proposed by Becke, taking into account the Bragg-Slater radius and the Becke's recipe suggesting to increase the radius of hydrogen to $0.35 \AA .{ }^{68}$ Each atom is integrated using Chebyshev's integration for the radial part and Lebedev's quadrature ${ }^{76}$ for the angular part.

\section{Results and discussion}

In our previous article, we presented evidence that the proportionality between the Fukui function and the local softness is also approximately true for the local hardness. In the literature several authors ${ }^{28,30-32,39,77,78}$ have shown that the Coulomb term of the hardness kernel is usually dominant in the evaluation of the local and global hardnesses, although the contribution from the kinetic energy is not negligible and can be comparable to the Coulombic term for some specific systems. ${ }^{25-27}$ If one considers only the Coulomb term, then it follows from eqn (5) and (14) that $\eta(\vec{r})$ and $\eta$ are equal to the Fukui function potential and the Fukui function electrostatic repulsion, ${ }^{45}$ respectively

$$
\begin{gathered}
\eta(\vec{r}) \cong \int \frac{f(\vec{r})}{\left|\vec{r}-\vec{r}^{\prime}\right|} \mathrm{d} \vec{r}^{\prime}=v_{f}(\vec{r}) \text { and } \\
\eta \cong \iint \frac{f(\vec{r}) f\left(\vec{r}^{\prime}\right)}{\left|\vec{r}-\vec{r}^{\prime}\right|} \mathrm{d} \vec{r} \mathrm{~d} \vec{r}^{\prime}=J_{f} .
\end{gathered}
$$

The local hardness and local softness are functions that measure the "local abundance" or "concentration" of the corresponding global properties. The difference resides that the integration of $s(\vec{r})$ will result in $S$, while the integration of $f(\vec{r}) \eta(\vec{r})$ will result in $\eta$. This fact can be seen, if one uses eqn (8), (14) and (1) in eqn (4)

$$
\int \eta(\vec{r}) s(\vec{r}) \mathrm{d} \vec{r}=\mathrm{S} \int \eta(\vec{r}) f(\vec{r}) \mathrm{d} \vec{r}=S \eta=1 .
$$

Thus it is not surprising that $\eta(\vec{r})$ and $s(\vec{r})$ contain the same potential "information" and their profiles can be similar. An interesting exception occurs at positions where $f(\vec{r})$ is zero. In this situation, $s(\vec{r})$ is also zero, see eqn (8), but the Fukui function potential and $\eta(\vec{r})$ may not be (see eqn (16)), and so they can be used to explain the regioselectivity of the system. For instance, along the $C_{6}$ axis of the benzene $\rho_{\mathrm{HOMO}}(\vec{r})$ and $\rho_{\text {LUMO }}(\vec{r})$ show null values, while the Fukui functions calculated with finite difference approximation, see eqn (9) and (10), and $\mathrm{s}(\vec{r})$ will be also zero or very small. In contrast, Fig. 1 shows that the two profiles of $\eta(\vec{r})$ exhibit maxima at the center of the ring and decrease smoothly along the $C_{6}$ axis. The $\eta(\vec{r})$ profiles have been calculated using $\rho_{\mathrm{HOMO}}(\vec{r})$ as approximation of $f(\vec{r})$ and two models of $F[(\vec{r})]$ : (a) the Coulombic Thomas-Fermi-1/9th Weizsäcker-Dirac-Wigner functional; (b) only the Coulomb term. As one can see, the Coulomb

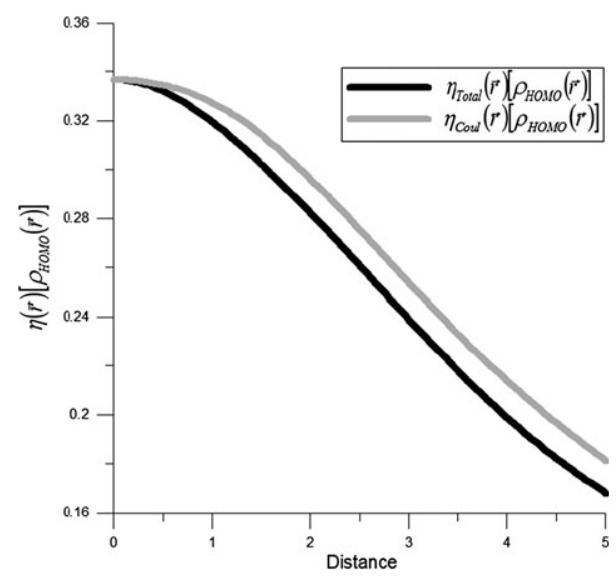

Fig. 1 Profile of local hardness along the $C_{6}$ axis of the benzene molecule at B3LYP/6-31 + G(d) level, with origin located at the centre of the ring. All values are given in a.u. 
term is dominant and the differences between the two $\eta(\vec{r})$ profiles of Fig. 1 originate from the contribution of the 1/9th Weizsäcker term, which depends on the gradient and Laplacian of the Fukui function. The other terms are proportional to $f(\vec{r})$ and they do not contribute to $\eta(\vec{r})$. This is an example of the non proportionality between $\eta(\vec{r})$ and $s(\vec{r})$, where $\eta(\vec{r})$ manifests itself as a more powerful descriptor than $s(\vec{r}){ }^{79}$

In conclusion it can be stated that the "chemical" (and traditional) interpretation of $s(\vec{r})$ and $\eta(\vec{r})$ as pointwise representations of the softest and hardest regions of a molecule is only valid if and only if $s(\vec{r})$ and $\eta(\vec{r})$ are only applied to soft and hard systems, respectively. It is worth noting that the majority of applications of these reactivity indices reported in the literature fulfil this requirement, although some of them can be interpreted and/or understood in a different way with this new perspective. In the forthcoming paragraphs, we will analyze in detail the implications of this perspective on the applicability of the local hardness and softness as reactivity descriptors and in the HSAB principle.

\section{(a) Implications as reactivity descriptors}

The large number of studies in the literature where $s(\vec{r})$ and $\eta(\vec{r})$ have been used makes it impossible to reinterpret all of them in terms of this new framework. To simplify this task, we will divide the analysis of the applicability of these descriptors into three cases, based on the location of the sites that are subjected to comparison:

(a) Two sites within one molecule.

(b) Two sites in two noninteracting molecules.

(c) Two sites in two interacting molecules.

A summary of these ideas can be found in Scheme 1-3, where the ordering of condensed atomic softness for different sites in one or two molecules is depicted and where an indication is given if relevant conclusions ("ok") or not $(\neq)$ can be drawn from comparing two sites. As we have commented before, $s(\vec{r})$ and $\eta(\vec{r})$ contain more or less the same potential information. Thus, the condensed atomic softness plotted in Scheme 1-3 can be interchanged with the condensed atomic hardness in the following analysis and conclusions will

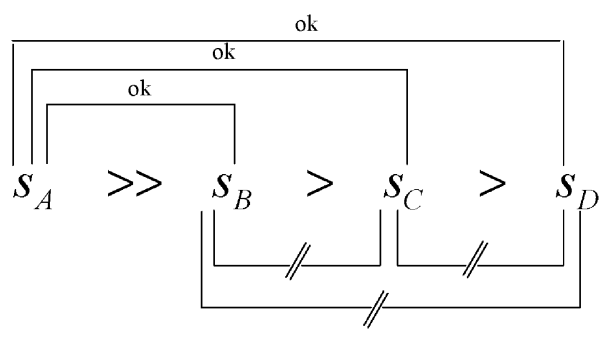

Scheme 1

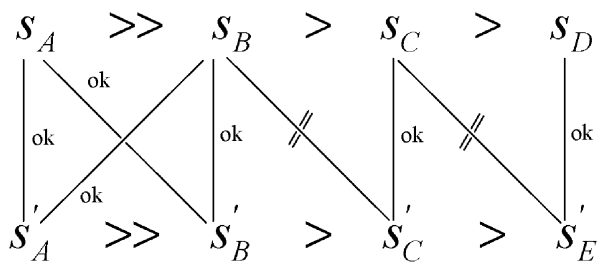

Scheme 2 still be valid. It is important to remark that the Scheme 1-3 are illustrative summaries of the possible allowed and forbidden links, which contain some redundant relationships (e.g. $S_{\mathrm{b}}>$ $S_{\mathrm{c}}$ and $S_{\mathrm{c}}>S_{\mathrm{d}}$ in Scheme 1) and where not necessarily all forbidden analyses have been written (e.g. $S_{c}$ and $S_{b}^{\prime}$ in Scheme 2).

Scheme 1 displays the permissible ("ok") and forbidden $(\neq)$ relationships that one can establish in the intramolecular analysis of local softness or condensed atomic softness. The local softness represents pointwise measures of the local abundance of the corresponding global softness. Thus, one can contrast the atom with by far the highest value of atomic softness, $s_{\mathrm{A}}$, with respect to the other regions of the molecule and $s_{\mathrm{A}}$ will represent a hard/soft site if the global softness of the molecule has a low/high value. However, one can not compare the much smaller condensed atomic softness (e.g. $s_{\mathrm{B}}$ vs. $s_{\mathrm{C}}, s_{\mathrm{B}} v s . s_{\mathrm{D}}, s_{\mathrm{C}} v s . s_{\mathrm{D}}$ ), because in those cases $s_{\mathrm{X}}$ no longer corresponds to the chemical concept of local softness and it only represents the atomic contribution to $S$. Consequently, in Scheme 1 one cannot say that $s_{\mathrm{B}}, s_{\mathrm{C}}$ and $s_{\mathrm{D}}$ symbolize the second, third, and fourth softest regions of the molecule, respectively.

Table 1 and 2 contain the global softness, global hardness, condensed local softness, and condensed atomic hardness for seven systems, which allow us to illustrate the conclusions derived from Scheme 1. The global hardness and softness of Table 1 have been evaluated using eqn (6) and (7) and $\eta\left[\rho_{\mathrm{HOMO}}(\vec{r})\right]$ and $\eta\left[\rho_{\text {LUMO }}(\vec{r})\right]$ obtained from the integration of the hardness kernel, see eqn (14) and (5), using $\rho_{\mathrm{HOMO}}(\vec{r})$ and $\rho_{\text {LUMO }}(\vec{r})$, respectively, as approximations of $f(\vec{r})$. In Table 1 one can see that $\eta\left[\rho_{\mathrm{HOMO}}(\vec{r})\right]$ and the hardness evaluated from eqn (6) and (7) give more or less the same tendencies. In contrast, $\eta\left[\rho_{\text {LUMO }}(\vec{r})\right]$ shows a different behaviour, e.g. it incorrectly predicts that methanol $(12.39 \mathrm{eV})$ and formaldehyde $(15.12 \mathrm{eV})$ are harder than water $(11.58 \mathrm{eV})$. This fact illustrates that $f^{-}(\vec{r})$ is a better approximation of the Fukui function than $f^{+}(\vec{r})$ because, if the exact Fukui function and Hohenberg-Kohn functional were used in eqn (14) the exact global hardness would be obtained. ${ }^{80}$ Moreover, several works $^{22,23,28}$ have shown that the global hardness using electrophilic Fukui functions, $f^{-}(\vec{r})$, yields a better reproduction of the experimental global hardness than with nucleophilic Fukui functions, $f^{+}(\vec{r})$. However, as $s^{+}(\vec{r})$ has been a typical reactivity descriptor for nucleophilic attacks, we want to show that the condensed atomic hardness of $\eta\left[\rho_{\text {LUMO }}(\vec{r})\right]$ contain the same potential information.

The values of Table 2 have to be considered as contributions to the corresponding global property. For instance, a value of $0.093 \mathrm{eV}^{-1}[19.11 \mathrm{eV}]$ in $s_{\mathrm{O}}^{-}\left[\eta\left[\rho_{\mathrm{HOMO}}(\vec{r})\right]\right]$ for methanol means that the oxygen atom contributes $71 \% \quad(=0.093 / 0.130 \times$ $100 \%)[87 \%(=19.11 / 22.03 \times 100 \%)]$ to the global softness [hardness] of the molecule. However, in the case of $s^{+}$ $\left[\eta\left[\rho_{\text {LUMO }}(\vec{r})\right]\right]$ the contribution of the carbon atom to the global softness of the methanol [hardness] can be similar or even higher (depending of the approximations used) than the oxygen atom. Therefore, it seems that $s^{-}\left[\eta\left[\rho_{\text {LUMO }}(\vec{r})\right]\right]$ and $s^{+}$ $\left[\eta\left[\rho_{\text {HOMO }}(\vec{r})\right]\right]$ can predict opposite results about the atomic contributions to the global softness [hardness]. This misunderstanding can be easily understood analyzing the implications 
Table 1 Global softness, $S$, and global hardness, $\eta$, for seven selected molecules evaluated at B3LYP/6-31+G(d,p) level. All units are eV

\begin{tabular}{|c|c|c|c|c|c|c|c|}
\hline & $S^{a}$ & $S^{b}$ & $\eta^{a}$ & $\eta^{b}$ & $\eta\left[\rho_{\text {НОМо }}(\vec{r})\right]^{c}$ & $\eta\left[\rho_{\mathrm{LUMO}}(\vec{r})\right]^{d}$ & $\eta_{\exp }^{e}$ \\
\hline $\mathrm{H}_{2} \mathrm{O}$ & 0.107 & 0.064 & 9.36 & 15.64 & 28.26 & 11.58 & 19.0 \\
\hline $\mathrm{CH}_{3} \mathrm{OH}$ & 0.130 & 0.081 & 7.68 & 12.36 & 22.03 & 12.39 & 17.0 \\
\hline $\mathrm{CH}_{3} \mathrm{OCH}_{3}$ & 0.134 & 0.087 & 7.47 & 11.51 & 19.78 & 8.48 & 16.0 \\
\hline $\mathrm{CH}_{2}=\mathrm{CHCHO}$ & 0.193 & 0.099 & 5.19 & 10.06 & 20.51 & 10.99 & $10.11^{f}$ \\
\hline $\mathrm{HCOH}$ & 0.168 & 0.085 & 5.96 & 11.72 & 20.78 & 15.12 & $10.88^{f}$ \\
\hline $\mathrm{CH}_{3} \mathrm{COH}$ & 0.160 & 0.086 & 6.24 & 11.59 & 18.10 & 17.42 & 10.22 \\
\hline $\mathrm{CH}_{3} \mathrm{COCH}_{3}$ & 0.159 & 0.090 & 6.28 & 11.13 & 19.95 & 14.52 & 9.70 \\
\hline
\end{tabular}

${ }^{a}$ Calculated with the frontier orbital approximation, eqn (7). ${ }^{b}$ Calculated with the Parr-Pearson approximation, eqn (6). ${ }^{c}$ Global hardness obtained from eqn (14) and (5) using $\rho_{\mathrm{HOMO}}(\vec{r})$ as approximation of $f(\vec{r}) .{ }^{d}$ Global hardness obtained from eqn (14) and (5) using $\rho_{\mathrm{LUMO}}(\vec{r})$ as approximation of $f(\vec{r}) .{ }^{e}$ Calculated with the Parr-Pearson approximation, eqn (6) using experimental values of ionization potential and electron affinity obtained from ref. 5,7,103. ${ }^{f}$ The experimental value of electron affinity is not available and the global hardness is approximated by the experimental value of the ionization potential obtained from ref. 103.

Table 2 Condensed local softness, $s_{\mathrm{X}}$, and condensed atomic hardness, $\eta_{\mathrm{X}}$, of oxygen and carbon atoms for the seven selected molecules in Table 1 evaluated at B3LYP/6-31+G(d,p) level. All units are eV

\begin{tabular}{|c|c|c|c|c|c|c|c|}
\hline & & $s_{\mathrm{X}}{ }^{a}$ & $s_{\mathrm{X}}^{-b}$ & $s_{\mathrm{X}}^{+c}$ & $s_{\mathrm{X}}^{+d}$ & $\eta_{\mathrm{X}}\left[\rho_{\mathrm{HOMO}}(\vec{r})\right]^{e}$ & $\eta_{\mathrm{X}}\left[\rho_{\text {LUMO }}(\vec{r})\right]^{f}$ \\
\hline $\mathrm{H}_{2} \mathrm{O}$ & $\mathrm{O}$ & 0.056 & 0.098 & 0.028 & 0.037 & 27.68 & 3.739 \\
\hline \multirow[t]{2}{*}{$\mathrm{CH}_{3} \mathrm{OH}$} & $\mathrm{C}$ & -0.005 & 0.013 & 0.043 & 0.018 & 1.196 & 1.032 \\
\hline & $\mathrm{O}$ & 0.049 & 0.093 & 0.016 & 0.027 & 19.11 & 1.963 \\
\hline \multirow[t]{2}{*}{$\mathrm{CH}_{3} \mathrm{OCH}_{3}$} & $\mathrm{C}$ & -0.006 & 0.009 & 0.029 & 0.013 & 0.728 & 0.585 \\
\hline & $\mathrm{O}$ & 0.047 & 0.085 & 0.006 & 0.008 & 16.55 & 0.524 \\
\hline \multirow[t]{4}{*}{$\mathrm{CH}_{2}=\mathrm{CHCHO}$} & $\mathrm{O}$ & 0.051 & 0.129 & 0.020 & 0.042 & 17.11 & 2.611 \\
\hline & $\mathrm{C}_{\text {carbonyl }}$ & -0.001 & 0.020 & 0.018 & 0.049 & 1.324 & 2.938 \\
\hline & $\mathrm{C}_{\alpha}$ & -0.002 & 0.017 & 0.008 & 0.029 & 0.880 & 1.468 \\
\hline & $\mathrm{C}_{\beta}$ & 0.019 & 0.002 & 0.033 & 0.062 & 0.061 & 3.580 \\
\hline \multirow[t]{2}{*}{$\mathrm{HCOH}$} & $\mathrm{O}$ & 0.048 & 0.112 & 0.022 & 0.058 & 17.12 & 5.747 \\
\hline & $\mathrm{C}_{\text {carbonyl }}$ & 0.004 & 0.019 & 0.056 & 0.094 & 1.419 & 8.562 \\
\hline \multirow{2}{*}{$\mathrm{CH}_{3} \mathrm{COH}$} & $\mathrm{O}$ & 0.046 & 0.105 & 0.020 & 0.053 & 16.52 & 5.465 \\
\hline & $\mathrm{C}_{\text {carbonyl }}$ & 0.002 & 0.017 & 0.048 & 0.082 & 1.369 & 8.759 \\
\hline \multirow[t]{2}{*}{$\mathrm{CH}_{3} \mathrm{COCH}_{3}$} & $\mathrm{O}$ & 0.046 & 0.104 & 0.020 & 0.049 & 16.43 & 4.791 \\
\hline & $\mathrm{C}_{\text {carbonyl }}$ & 0.004 & 0.016 & 0.026 & 0.073 & 1.287 & 8.140 \\
\hline
\end{tabular}

${ }^{a}$ From eqn (11) and (9), where the softness is evaluated using eqn (6) and the condensed Fukui function using NPA charges. ${ }^{b}$ From eqn (11) and (9), where the softness is evaluated using eqn (7) and the condensed Fukui function using the integration over the Voronoi polyhedra of $\rho_{\text {Номо } \vec{r} .}{ }^{c}$ From eqn (11) and (10), where the softness is evaluated using eqn (6) and the condensed Fukui function using NPA charges. ${ }^{d}$ From eqn (11) and (10), where the softness is evaluated using eqn (7) and the condensed Fukui function using the integration over the Voronoi polyhedra of $\rho_{\text {LUMO }}(\vec{r}) .{ }^{e}$ Condensed atomic hardness obtained from the numerical integration of eqn (15) and (5) using $\rho_{\text {HOMO }}(\vec{r})$ as approximation of $f(\vec{r}) .{ }^{f}$ Condensed atomic hardness obtained from the numerical integration of eqn (15) and (5) using $\rho_{\text {Lumo }}(\vec{r})$ as approximation of $f(\vec{r})$.

of the finite difference in $S$ and $\eta \cdot s^{-}\left[\eta\left[\rho_{\mathrm{HOMO}}(\vec{r})\right]\right]$ represents the local abundance of the global softness [hardness] resulting from subtracting one electron, while $s^{+}\left[\eta\left[\rho_{\text {LUMO }}(\vec{r})\right]\right]$ represents the local abundance of the global softness [hardness] resulting from adding one electron. In most of the systems, the left derivative ( $I$ and $\varepsilon_{\text {HOMO }}$ ) contributions to $S$ and $\eta$ is larger than the right derivative ( $A$ and $\varepsilon_{\text {LUMO }}$ ) contributions. For this reason, $s^{-}$and $\eta\left[\rho_{\mathrm{HOMO}}(\vec{r})\right]$ are better representations of the concentration of $S$ and $\eta$ and it explains their different behaviours with respect to $s^{+}$and $\eta\left[\rho_{\text {LUMO }}(\vec{r})\right]$.

In the case of acrolein, $\mathrm{CH}_{2}=\mathrm{CHCHO}, s_{\mathrm{O}}^{-}$and $\eta_{\mathrm{o}}\left[\rho_{\mathrm{HOMO}}(\vec{r})\right]$ show the largest values, indicating that $S$ and $\eta$ are mainly due to the oxygen atom. However, as we have seen in Scheme 1, we cannot declare which is the second softest region of the molecule. On the other hand, $s_{\mathrm{X}}^{+}$and $\eta_{\mathrm{X}}\left[\rho_{\text {LUMO }}(\vec{r})\right]$ show quite similar values for the oxygen and the three carbon atoms, indicating that the local abundance of $S$ and $\eta$, respectively, resulting from adding one electron to acrolein is more or less the same for the four atoms. Therefore, we cannot say that the largest one, $C_{\beta}$, is the softest region of the molecule. However, we can declare that a nucleophilic attack on acrolein will be on the atom, which will show the largest redistribution of the electron density, i.e. with the largest $f_{\mathrm{X}}^{+}, s_{\mathrm{X}}^{+}$, and $\eta_{\mathrm{X}}\left[\rho_{\mathrm{LUMO}}(\vec{r})\right]$ values.

The next step is to analyze the applicability of these descriptors for comparing the reactivity of different molecules. For instance, when an atom or a group is replaced by a different one, what is the effect on the global hardness and the reactivity of the system? A first requirement is that the original and new systems have to show similar global hardnesses. Otherwise it can happen that for two sites from different systems, both with high $s(\vec{r})$ (i.e. a high value of $f(\vec{r})$ ), the first region can represent a soft region (large value of $S$ ), while the second represents a hard region (small value of $S$ ). For this reason when we study a family of molecules we cannot replace the atom(s) or group(s) which mainly contribute to the global property. In these conditions, we can, for example, analyze the effect of a new substituent on the global hardness and softness and on the atomic contributions of these properties for the atoms surrounding this new substituent. A summary of these ideas is displayed in the Scheme 2, where it is worth nothing that not all forbidden analyses have been written. 
Table 1 and 2 contain the $\mathrm{H}_{2} \mathrm{O}, \mathrm{CH}_{3} \mathrm{OH}$, and $\mathrm{CH}_{3} \mathrm{OCH}_{3}$ family, where the replacement of $\mathrm{H}$ for $\mathrm{CH}_{3}$ (an electron donor group) reduces [increases] the global hardness [softness]. This inductive effect makes that oxygen atoms of $\mathrm{CH}_{3} \mathrm{OH}$ and $\mathrm{CH}_{3} \mathrm{OCH}_{3}$ richer in electrons (and easier to ionize) than the oxygen atom of the water molecule. Then, the contribution of these atoms to the global properties will show the following tendencies: $s_{\mathrm{O}}^{-}\left(\mathrm{H}_{2} \mathrm{O}\right)>s_{\mathrm{O}}^{-}\left(\mathrm{CH}_{3} \mathrm{OH}\right)>s_{\mathrm{O}}^{-}\left(\mathrm{CH}_{3} \mathrm{OCH}_{3}\right)$ and $\eta_{\mathrm{O}}\left[\rho_{\mathrm{HOMO}}(\vec{r})\right]\left(\mathrm{H}_{2} \mathrm{O}\right)>\eta_{\mathrm{O}}\left[\rho_{\mathrm{HOMO}}(\vec{r})\right]\left(\mathrm{CH}_{3} \mathrm{OH}\right)>$ $\eta_{\mathrm{O}}\left[\rho_{\mathrm{HOMO}}(\vec{r})\right]\left(\mathrm{CH}_{3} \mathrm{OCH}_{3}\right)$.

One of the classical examples in the literature to show the utility of the Fukui function, local softness, and local hardness towards a nucleophilic attack has been the carbonyl compounds. ${ }^{32,81-88}$ In Table 1, one can see that the replacement of the $\mathrm{H}$ for $\mathrm{CH}_{3}$ in the formaldehyde, acetaldehyde, and acetone molecules also provokes a decrease of the global hardness, a tendency that is only reproduced from our calculations using the approximation of eqn (6). As we have already seen in the carbonyl compounds, the global hardness and softness are mainly due to the oxygen atom, which is described by $s_{\mathrm{X}}$ and $\eta_{\mathrm{X}}\left[\rho_{\mathrm{HOMO}}(\vec{r})\right]$. It is curious to remark that $\eta_{\mathrm{O}}\left[\rho_{\mathrm{HOMO}}(\vec{r})\right]$ predicts the tendency of the experimental global hardness, in contrast to $\eta\left[\rho_{\mathrm{HOMO}}(\vec{r})\right]$. In addition, the reactivity of these compounds is characterized by nucleophilic attacks and by $s_{\mathrm{X}}^{+}$and $\eta_{\mathrm{X}}\left[\rho_{\mathrm{LUMO}}(\vec{r})\right]$ indices. In Table 2 , one can see that the two ways to evaluate $s_{\mathrm{C}}^{+}$reproduce the correct reactivity of the carbon atom of the carbonyl group with respect to a nucleophilic attack (formaldehyde $>$ acetaldehyde $>$ acetone). On the other hand, $\eta_{C}\left[\rho_{\text {LUMO }}(\vec{r})\right]$ fails in this prediction and it follows the same trend that $\eta\left[\rho_{\text {LUMO }}(\vec{r})\right]$. In fact, it is difficult to consider that the condensed versions of the local softness and hardness can be useful to predict the reactivity if the global counterparts cannot reproduce the experimental values of the global hardness. Nevertheless we consider that they can be powerful reactivity descriptors if they are applied in the optimum conditions.

Finally, we will analyze the utility of these descriptors to describe the interaction between two different systems, see Scheme 3. Several studies have been reported about this topic, mainly focused on cycloadditions reactions. ${ }^{48,89-97}$ To illustrate our point of view we will use a normal electron demand Diels-Alder reaction, where the diene (we consider the diene in cis form) and dienophile contain an electron-donating (tert-butyl group) and electron-withdrawing (carboxylic group) groups, respectively, see Fig. 2. The first requirement is that the two systems have to show a similar global hardness, i.e. 5.307 and $6.133 \mathrm{eV}$ for the diene and dienophile, respectively, values obtained from eqn (7). The second requirement is that the atoms that one compares are the main

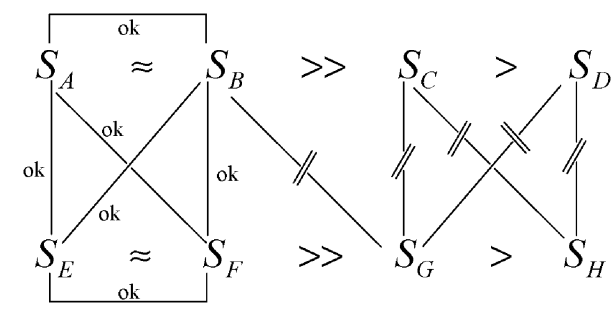

Scheme 3
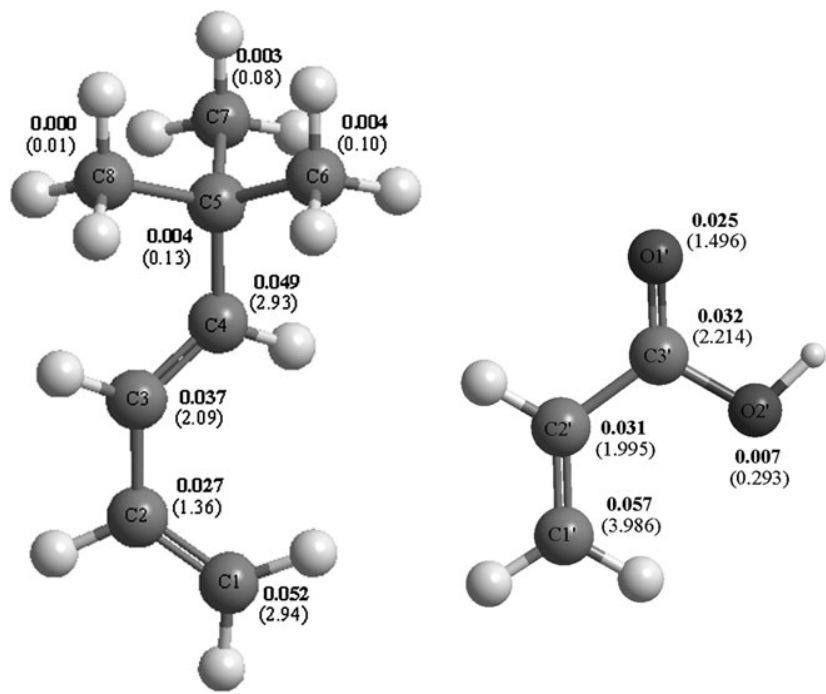

Fig. 2 Diels-Alder between a diene and dienophole with electrondonating and electron-withdrawing groups, respectively. The bold numbers of the diene and dienophile molecules are the condensed local softness from eqn (12), where the softness is evaluated using eqn (7) and the condensed Fukui function using the integration over the Voronoi polyhedral of $\rho_{\mathrm{HOMO}}(\vec{r})$ and $\rho_{\text {LUMO }}(\vec{r})$, respectively. The numbers between brackets of the diene and dienophile molecules are condensed atomic hardness obtained from the numerical integration of eqn (15) and (5) using $\rho_{\mathrm{HOMO}}(\vec{r})$ and $\rho_{\text {LUMO }}(\vec{r})$, respectively, as approximation of $f(\vec{r})$.

contributions to the global hardness and softness. For instance, in Fig. 2 the atomic contribution of $\mathrm{C} 1$ and $\mathrm{C} 4$ of the diene represent more than the $50 \%$ of the global hardness and softness of the system (the same happens to $\mathrm{C}^{\prime}$ and $\mathrm{C}^{\prime}$ of the dienophile). The regioselectivity will be controlled by the largest contributions of the systems, i.e. $\mathrm{C} 1$ of the diene and $\mathrm{Cl}^{\prime}$ of the dienophile, yielding the ortho cycloadduct.

Notice that the comparison is between the largest values of $s_{i, \text { diene }}^{-}\left[\eta_{x}\right.$,diene $\left.\left[\rho_{\mathrm{HOMO}}(\vec{r})\right]\right]$ and the largest values of $s_{i, \text { dienophile }}^{+}$ $\left[\eta_{x, \text { dienophile }}\left[\rho_{\text {LUMO }}(\vec{r})\right]\right]$. This fact implies the comparison of the regions which contribute more to the global softness [hardness] of the diene and dienophile in the cases of electron subtraction and electron addition, respectively. Then we are not comparing the two softest regions of the molecule, because otherwise we need to compare $s_{i, \text { diene }}^{-}\left[\eta_{x, \text { diene }}\left[\rho_{\text {HOMO }}(\vec{r})\right]\right]$ with respect to $s_{i \text {,dienophile }}^{-}\left[\eta_{x \text {,dienophile }}\left[\rho_{\mathrm{HOMO}}(\vec{r})\right]\right]$. This point of view is aligned with the idea proposed by Gázquez and Méndez that "the interaction between two molecules A and B will not necessary occur through the softer atoms but through those whose Fukui functions are approximately equal" as opposed to "the interaction between A and B is favoured when it occurs through those atoms whose softnesses are approximately equal". ${ }^{46}$ In fact, the same values of Fukui function imply the same contributions to the global properties.

\section{(b) Implications for the HSAB principle}

In this section we will firstly analyze the controversy about the hard-hard interactions, whether they are better described with maxima or with minima of $f(\vec{r})$ and how this new understanding of $s(\vec{r})$ and $\eta(\vec{r})$ can provide us a clear and elegant 
solution to this topic. In addition, the conclusions derived from this study will bring us a new point of view to the applicability of the HSAB principle and the global hardness and softness.

Melin et al. ${ }^{52}$ studied the protonation reaction of hydroxylamine $\left(\mathrm{NH}_{2} \mathrm{OH}\right)$ and thiohydroxylamine $\left(\mathrm{NH}_{2} \mathrm{SH}\right)$ systems using different condensed Fukui functions and charges. Experimental results ${ }^{98}$ show that in these two amines the nitrogen is the most susceptible site to be protonated. According to the rule of $\mathrm{Li}$ and Evans ${ }^{47}$ that the hard reactions tend to occur at the site with smallest Fukui functions, i.e. the smallest local softnesses, the protonation of $\mathrm{NH}_{2} \mathrm{OH}$ and $\mathrm{NH}_{2} \mathrm{SH}$ must to be on the oxygen and nitrogen atoms, respectively, see Fig. 3a and $4 \mathrm{a}$. For this reason Melin et al. ${ }^{52}$ proposed that a better description of the preferred site of protonation reaction is obtained with the charges than the Fukui functions.

The $s(\vec{r})$ and $\eta(\vec{r})$ are pointwise measures of the local abundance of $S$ and $\eta$, respectively. Thus, the only information that one can obtain from Fig. 3 and 4 is that the global hardness and softness of $\mathrm{NH}_{2} \mathrm{OH}$ is mainly due to the nitrogen atom, while the sulfur is the responsible for $\eta$ and $S$ of $\mathrm{NH}_{2} \mathrm{SH}$. In fact, the operational equations of the global hardness and softness, see eqn (6) and (7), can be more or less considered as molecular orbital-controled, because they involve first vertical ionization potential and electron affinity and the energies of the HOMO and LUMO. Then, it is clear that $s(\vec{r})$ and $\eta(\vec{r})$ can only predict orbital-controlled reactions and they cannot be used to predict the protonation site of these amines. Moreover, we can affirm that $s(\vec{r})$ and $\eta(\vec{r})$ can not describe any charge-controlled process because they have not been designed to explain these reactions.

Another example of this situation can be seen in Fig. 5, which contains the plots of $s^{-}(\vec{r})$ and $\eta(\vec{r})\left[\rho_{\mathrm{HOMO}}(\vec{r})\right]$ for formic acid. The deformation of the electronic cloud of formic acid upon accepting or donating electrons involves mainly the carbon and oxygen atoms of the carbonyl group; and the $\eta$ and $S$ and their local abundances have to represent this picture, as confirmed by Fig. 5 . Then, we cannot expect $s(\vec{r})$ and $\eta(\vec{r})$ to explain the acidity of this carboxylic acid, which is another charge-controlled process.

It is worth noting that these results complement the idea that $s(\vec{r})$ and $\eta(\vec{r})$ contain the same potential information and as we have already seen they have important implications in the HSAB principle, which can be summarized in the following conclusions:

(a) For orbital-controlled cases of the HSAB type, the extent of electron transfer can be described by $\eta$ and $S$

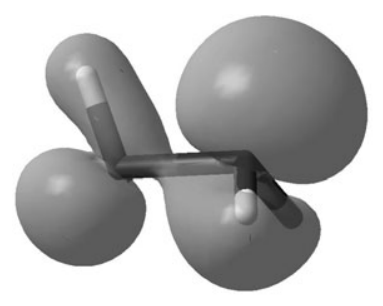

a)

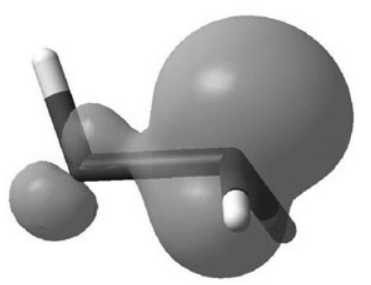

b)
Fig. 3 Three-dimensional contour plots of (a) $s^{-}(\vec{r})(0.01$ a.u.) and (b) $\eta(\vec{r})\left[\rho_{\mathrm{HOMO}}(\vec{r})\right] \quad(0.5$ a.u. $)$ for the hydroxylamine molecule at B3LYP $/ 6-31+\mathrm{G}(\mathrm{d})$ level.

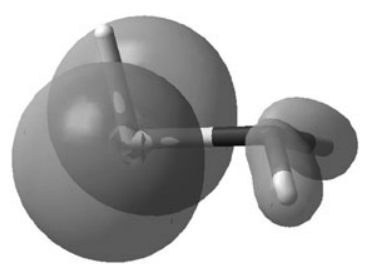

a)

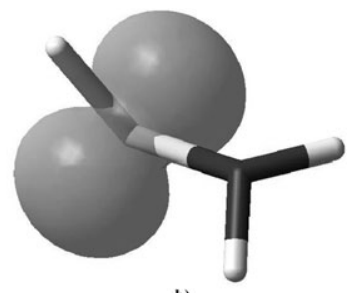

b)
Fig. 4 Three-dimensional contour plots of (a) $s^{-}(\vec{r})(0.01$ a.u.) and (b) $\eta(\vec{r})\left[\rho_{\mathrm{HOMO}}(\vec{r})\right](0.5$ a.u. $)$ for the thiohydroxylamine molecule at B3LYP/6-31 + G(d) level.

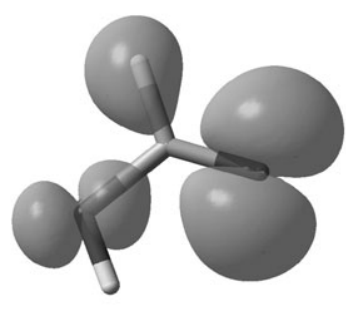

a)

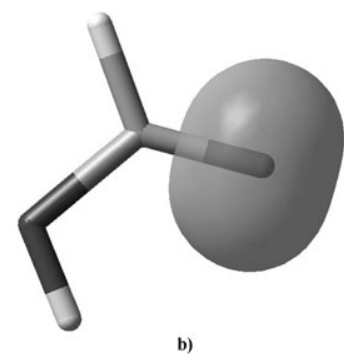

b)
Fig. 5 Three-dimensional contour plots of (a) $s^{-}(\vec{r})(0.01$ a.u.) and (b) $\eta(\vec{r})\left[\rho_{\mathrm{HOMO}}(\vec{r})\right](0.5$ a.u.) for formic acid at B3LYP/6-31+G(d) level.

and the regioselectivity can be described by either of the corresponding concentration descriptors, $\eta(\vec{r})$ or $s(\vec{r})$.

(b) Orbital-controlled HSAB interactions will occur at the maxima of the Fukui function whether they are soft-soft or hard-hard interactions.

(c) If a reaction is a charge-controlled, it cannot be described by $\eta, S, \eta(\vec{r})$, and $s(\vec{r})$ as descriptors and it cannot be discussed on the basis of the HSAB principle.

The fact that $\eta$ and $S$ are purely electronic properties and only appropriate for orbital-controlled interactions (remember that their operational equations, see eqn (6) and (7), involve first vertical ionization potential and electron affinity and the energies of the HOMO and LUMO) and the request that $\eta$ fits into the HSAB principle (the global hardness has to describe all the processes involved in the HSAB principle) has an important effect on the applicability of this principle, because it eliminates the role of electrostatic interactions.

Another possible solution of this problem is to go back to the Pearson's idea that hard-hard charge-controlled reactions are also included in the HSAB principle. It has been observed, ${ }^{2,14,99,100}$ and theoretically justified, ${ }^{101}$ that the hardness correlates with molecular size and charge, and in this sense the hardness can be also pertinent to chargecontrolled reactions. In this scheme, just the (c) conclusion shows minimal changes:

(c') If a reaction is a charge-controlled, $\eta, S, \eta(\vec{r})$, and $s(\vec{r})$ do not play a decisive role in the reactivity. Electrostatic effects are dominant in such cases, ${ }^{6,52,101}$ except for the (very exceptional) tie-breaker cases $^{53}$ where there are equivalent electrostatic sites. ${ }^{102}$

When one includes charge-controlled reactions (and possibly also polarizability-controlled reactions) in the HSAB principle, then linear combinations of reactivity descriptors naturally arise. $^{37,53-55}$ However, it is important to remark that (c) 
indicates that Parr-Pearson definition of global hardness in eqn (1) is incomplete, because it doesn't fully include the electrostatic effects. Including charge-controlled reactions in the HSAB forces one to consider redefining the hardness in a way that accommodates the expanded scope of the concept. It is impossible to state which interpretation of the HSAB principle is "better" based on the results of this paper and further work is certainly warranted.

\section{Acknowledgements}

M. T. thanks the European Community for financial help through the postdoctoral grant MEIF-CT-2006-025362. P. G. and F. D. $P$ are indebted to the Fund for Scientific Research-Flanders (FWO) and to the Free University of Brussels for continuous support to their group. P. W. A. thanks NSERC, the Canada Research Chairs, and Sharcnet for research support.

\section{References}

1 R. G. Pearson, J. Am. Chem. Soc., 1963, 85, 3533-3543.

2 R. G. Pearson, Science, 1966, 151, 172-176.

3 R. G. Pearson, J. Chem. Educ., 1968, 45, 581-587.

4 R. G. Pearson, J. Chem. Educ., 1968, 45, 643-648.

5 R. G. Pearson, Chemical Hardness: Applications from Molecules to Solids, Wiley-VCH, Oxford, 1997.

6 P. W. Ayers, R. G. Parr and R. G. Pearson, J. Chem. Phys., 2006, 124, 194107.

7 R. G. Parr and W. Yang, Density-Functional Theory of Atoms and Molecules, Oxford University Press, New York, 1989.

8 H. Chermette, J. Comput. Chem., 1999, 20, 129-154.

9 P. Geerlings, F. De Proft and W. Langenaeker, Chem. Rev., 2003, 103, 1793-1873.

10 P. Geerlings and F. De Proft, Phys. Chem. Chem. Phys., 2008, 10, 3028-3042.

11 P. W. Ayers, J. S. M. Anderson and L. J. Bartolotti, Int. J. Quantum Chem., 2005, 101, 520-534.

12 J. L. Gázquez, J. Mex. Chem. Soc., 2008, 52, 3-10.

13 S. B. Liu, Acta Phys.-Chim. Sinica, 2009, 25, 590-600.

14 R. G. Parr and R. G. Pearson, J. Am. Chem. Soc., 1983, 105, $7512-7516$.

15 W. T. Yang and R. G. Parr, Proc. Natl. Acad. Sci. U. S. A., 1985, 82, 6723-6726.

16 S. K. Ghosh and M. Berkowitz, J. Chem. Phys., 1985, 83, 2976-2983.

17 M. Berkowitz, S. K. Ghosh and R. G. Parr, J. Am. Chem. Soc., 1985, 107, 6811-6814.

18 M. K. Harbola, P. K. Chattaraj and R. G. Parr, Isr. J. Chem., 1991, 31, 395-402.

19 P. K. Chattaraj, D. R. Roy, P. Geerlings and M. TorrentSucarrat, Theor. Chem. Acc., 2007, 118, 923-930.

20 P. W. Ayers and R. G. Parr, J. Chem. Phys., 2008, 128, 184108.

21 J. Garza and J. Robles, Int. J. Quantum Chem., 1994, 49, 159-169.

22 M. Torrent-Sucarrat, M. Duran and M. Solà, J. Phys. Chem. A, 2002, 106, 4632-4638.

23 M. Torrent-Sucarrat, J. M. Luis, M. Duran and M. Solà, J. Mol. Struct. (THEOCHEM), 2005, 727, 139-148.

24 S. K. Ghosh, Chem. Phys. Lett., 1990, 172, 77-82.

25 M. Torrent-Sucarrat and P. Geerlings, J. Chem. Phys., 2006, 125, 244101.

26 M. Torrent-Sucarrat, P. Salvador, P. Geerlings and M. Solà, J. Comput. Chem., 2007, 28, 574-583.

27 M. Torrent-Sucarrat, P. Salvador, M. Solà and P. Geerlings, J. Comput. Chem., 2008, 29, 1064-1072.

28 S. B. Liu, F. De Proft and R. G. Parr, J. Phys. Chem. A, 1997, 101, 6991-6997.

29 J. L. Gázquez, in Structure and Bonding, ed. K. D. Sen, 1993, vol. 80 , p. 27.

30 S. Saha and R. K. Roy, J. Phys. Chem. B, 2007, 111, 9664-9674.
31 S. Saha and R. K. Roy, J. Phys. Chem. B, 2008, 112, 1884-1884. 32 S. Saha and R. K. Roy, Phys. Chem. Chem. Phys., 2008, 10, 5591-5598.

33 P. Mignon, P. Geerlings and R. A. Schoonheydt, J. Phys. Chem. C, 2007, 111, 12376-12382.

34 A. S. Ozen, F. De Proft, V. Aviyente and P. Geerlings, J. Phys. Chem. A, 2006, 110, 5860-5868.

35 P. Mignon, S. Loverix and P. Geerlings, Chem. Phys. Lett., 2005, 401, 40-46.

36 A. Olasz, P. Mignon, F. De Proft, T. Veszpremi and P. Geerlings, Chem. Phys. Lett., 2005, 407, 504-509.

37 W. Langenaeker, F. De Proft and P. Geerlings, J. Phys. Chem., 1995, 99, 6424-6431.

38 P. Mignon, S. Loverix, J. Steyaert and P. Geerlings, Nucleic Acids Res., 2005, 33, 1779-1789.

39 A. Borgoo, M. Torrent-Sucarrat, F. De Proft and P. Geerlings, J. Chem. Phys., 2007, 126, 234104.

40 P. W. Ayers and R. G. Parr, J. Am. Chem. Soc., 2000, 122, 2010-2018.

41 We have focused on the frontier local hardness, because it is not possible to evaluate the unconstrained local hardness and the total local hardness has been subject to criticism, when it is used to evaluate global hardness. For more details see ref. 19.

42 M. Berkowitz and R. G. Parr, J. Chem. Phys., 1988, 88, 2554-2557.

43 M. Torrent-Sucarrat, F. De Proft, P. Geerlings and P. W. Ayers, Chem.-Eur. J., 2008, 14, 8652-8660.

44 G. Klopman, J. Am. Chem. Soc., 1968, 90, 223-234.

45 M. Berkowitz, J. Am. Chem. Soc., 1987, 109, 4823-4825.

46 J. L. Gázquez and F. Méndez, J. Phys. Chem., 1994, 98 , 4591-4593.

47 Y. Li and J. N. S. Evans, J. Am. Chem. Soc., 1995, 117, $7756-7759$.

48 S. Damoun, G. Van de Woude, F. Méndez and P. Geerlings, J. Phys. Chem. A, 1997, 101, 886-893.

49 Y. Li and J. N. S. Evans, Proc. Natl. Acad. Sci. U. S. A., 1996, 93, $4612-4616$.

50 P. Pérez, Y. Simon-Manso, A. Aizman, P. Fuentealba and R. Contreras, J. Am. Chem. Soc., 2000, 122, 4756-4762.

51 P. K. Chattaraj, J. Phys. Chem. A, 2001, 105, 511-513.

52 J. Melin, F. Aparicio, V. Subramanian, M. Galvan and P. K. Chattaraj, J. Phys. Chem. A, 2004, 108, 2487-2491.

53 J. S. M. Anderson, J. Melin and P. W. Ayers, J. Chem. Theory Comput., 2007, 3, 358-374.

54 J. S. M. Anderson and P. W. Ayers, Phys. Chem. Chem. Phys., 2007, 9, 2371-2378.

55 J. S. M. Anderson, J. Melin and P. W. Ayers, J. Chem. Theory Comput., 2007, 3, 375-389.

56 T. Koopmans, Physica, 1934, 1, 104-113.

57 R. G. Parr and W. T. Yang, J. Am. Chem. Soc., 1984, 106, 4049-4050.

58 P. W. Ayers and M. Levy, Theor. Chem. Acc., 2000, 103, 353-360.

59 K. Fukui, T. Yonezawa and H. Shingu, J. Chem. Phys., 1952, 20, $722-725$.

60 K. Fukui, T. Yonezawa, C. Nagata and H. Shingu, J. Chem. Phys., 1954, 22, 1433-1442.

61 W. Yang and W. J. Mortier, J. Am. Chem. Soc., 1986, 108, 5708-5711.

62 R. S. Mulliken, J. Comput. Chem., 1955, 23, 1833-1841.

63 A. E. Reed, L. A. Curtiss and F. Weinhold, Chem. Rev., 1988, 88, 899-926.

64 F. Gilardoni, J. Weber, H. Chermette and T. R. Ward, J. Phys. Chem. A, 1998, 102, 3607-3613.

65 F. A. Bulat, E. Chamorro, P. Fuentealba and A. Toro-Labbé, J. Phys. Chem. A, 2004, 108, 342-349.

66 R. F. W. Bader, Atoms in Molecules: A Quantum Theory, Clarendon, Oxford, 1990

67 F. L. Hirshfeld, Theor. Chim. Acta, 1977, 44, 129-138.

68 A. D. Becke, J. Chem. Phys., 1988, 88, 2547-2553.

69 In ref. 18, Harbola, Chattaraj, and Parr show that when this definition of the local hardness is computed exactly, the local hardness is a constant. This does not contradict the findings of this paper, however, because while we are using accurate approximations to the ground-state density and the Fukui function, our model for the hardness kernel is only qualitatively correct. 
70 W. J. Hehre, R. Ditchfield and J. A. Pople, J. Chem. Phys., 1972, 56, 2257-2261.

71 W. J. Hehre, L. Radom, P. v. R. Schleyer and J. A. Pople, Ab initio Molecular Orbital Theory, Wiley, New York, 1986.

72 A. D. Becke, J. Chem. Phys., 1993, 98, 5648-5652.

73 C. T. Lee, W. T. Yang and R. G. Parr, Phys. Rev. B: Condens. Matter, 1988, 37, 785-789.

74 M. J. Frisch, G. W. Trucks, H. B. Schlegel, G. E. Scuseria, M. A. Robb, J. R. Cheeseman, J. A. Montgomery, Jr., T. Vreven, K. N. Kudin, J. C. Burant, J. M. Millam, S. S. Iyengar, J. Tomasi, V. Barone, B. Mennucci, M. Cossi, G. Scalmani, N. Rega, G. A. Petersson, H. Nakatsuji, M. Hada, M. Ehara, K. Toyota, R. Fukuda, J. Hasegawa, M. Ishida, T. Nakajima, Y. Honda, O. Kitao, H. Nakai, M. Klene, X. Li, J. E. Knox, H. P. Hratchian, J. B. Cross, V. Bakken, C. Adamo, J. Jaramillo, R. Gomperts, R. E. Stratmann, O. Yazyev, A. J. Austin, R. Cammi, C. Pomelli, J. Ochterski, P. Y. Ayala, K. Morokuma, G. A. Voth, P. Salvador, J. J. Dannenberg, V. G. Zakrzewski, S. Dapprich, A. D. Daniels, M. C. Strain, O. Farkas, D. K. Malick, A. D. Rabuck, K. Raghavachari, J. B. Foresman, J. V. Ortiz, Q. Cui, A. G. Baboul, S. Clifford, J. Cioslowski, B. B. Stefanov, G. Liu, A. Liashenko, P. Piskorz, I. Komaromi, R. L. Martin, D. J. Fox, T. Keith, M. A. Al-Laham, C. Y. Peng, A. Nanayakkara, M. Challacombe, P. M. W. Gill, B. G. Johnson, W. Chen, M. W. Wong, C. Gonzalez and J. A. Pople, GAUSSIAN 03, Gaussian, Inc., Wallingford, CT, 2003.

75 M. Torrent-Sucarrat, S. B. Liu and F. De Proft, J. Phys. Chem. A, 2009, 113, 3698-3702.

76 V. I. Levedev and D. N. Laikov, Dokl. Math., 1999, 59, 477.

77 P. K. Chattaraj, A. Cedillo and R. G. Parr, J. Chem. Phys., 1995, 103, 10621-10626.

78 L. F. Pacios, Chem. Phys. Lett., 1997, 276, 381-387.

79 M. Torrent-Sucarrat, S. De Romagnoli and P. Geerlings, unpublished results.

80 P. K. Chattaraj, A. Cedillo and R. G. Parr, J. Chem. Phys., 1995, 103, 7645-7646.

81 P. Mondal, K. K. Hazarika and R. C. Deka, PhysChemComm, 2003, 6, 24-27.

82 F. Méndez and J. L. Gázquez, J. Am. Chem. Soc., 1994, 116, 9298-9301.
83 R. K. Roy, F. De Proft and P. Geerlings, J. Phys. Chem. A, 1998, 102, 7035-7040.

84 S. Krishnamurty and S. Pal, J. Phys. Chem. A, 2000, 104, 7639-7645.

85 F. Nazari and F. R. Zali, J. Mol. Struct. (THEOCHEM), 2007, 817, 11-18.

86 R. K. Roy, J. Phys. Chem. A, 2004, 108, 4934- 4939.

87 R. K. Roy, K. Choho, F. De Proft and P. Geerlings, J. Phys. Org. Chem., 1999, 12, 503-509.

88 R. K. Roy, N. Tajima and K. Hirao, J. Phys. Chem. A, 2001, 105, 2117-2124.

89 Y. Canac, N. Debono, L. Vendier and R. Chauvin, Inorg. Chem., 2009, 48, 5562-5568.

90 A. K. Chandra and M. T. Nguyen, J. Phys. Chem. A, 1998, 102, 6181-6185.

91 A. K. Chandra and M. T. Nguyen, J. Comput. Chem., 1998, 19, 195-202.

92 S. Krishnamurty, R. K. Roy, R. Vetrivel, S. Iwata and S. Pal, J. Phys. Chem. A, 1997, 101, 7253-7257.

93 G. Molteni and A. Ponti, Tetrahedron, 2003, 59, 5225-5229.

94 L. T. Nguyen, F. De Proft, V. L. Dao, M. T. Nguyen and P. Geerlings, J. Phys. Org. Chem., 2003, 16, 615-625.

95 L. T. Nguyen, T. N. Le, F. De Proft, A. K. Chandra, W. Langenaeker, M. T. Nguyen and P. Geerlings, J. Am. Chem. Soc., 1999, 121, 5992-6001.

96 A. Ponti and G. Molteni, J. Org. Chem., 2001, 66, 5252-5255.

97 A. Ponti, J. Phys. Chem. A, 2000, 104, 8843-8846.

98 F. Angelelli, M. Aschi, F. Cacace, F. Pepi and G. Depetris, J. Phys. Chem., 1995, 99, 6551-6556.

99 T. K. Ghanty and S. K. Ghosh, J. Phys. Chem., 1996, 100, 17429-17433.

100 T. K. Ghanty and S. K. Ghosh, J. Phys. Chem., 1993, 97, 4951-4953.

101 P. W. Ayers, Faraday Discuss., 2007, 135, 161-190.

102 The conclusions $\mathrm{a}, \mathrm{b}, \mathrm{c}$, and $\mathrm{c}^{\prime}$ have been obtainedconsidering the definition of the local hardness and local softness provided by the eqn (5) and (8), respectively. However, it is important to remark that there exist different ways to evaluate the local hardness, which can result to different conclusions to the charge-controlled processes.

103 NIST Standard Reference Data Program, http://webbook.nist.gov/ chemistry/form-ser.html. 